\title{
Active demethylation in mouse zygotes involves cytosine deamination and base excision repair
}

\author{
Fátima Santos ${ }^{1 *}$, Julian Peat ${ }^{1}$, Heather Burgess ${ }^{1,3}$, Cristina Rada ${ }^{2}$, Wolf Reik ${ }^{1,3}$ and Wendy Dean ${ }^{1}$
}

\begin{abstract}
Background: DNA methylation in mammals is an epigenetic mark necessary for normal embryogenesis. During development active loss of methylation occurs in the male pronucleus during the first cell cycle after fertilisation. This is accompanied by major chromatin remodelling and generates a marked asymmetry between the paternal and maternal genomes. The mechanism(s) by which this is achieved implicate, among others, base excision repair (BER) components and more recently a major role for TET3 hydroxylase. To investigate these methylation dynamics further we have analysed DNA methylation and hydroxymethylation in fertilised mouse oocytes by indirect immunofluorescence (IF) and evaluated the relative contribution of different candidate factors for active demethylation in knock-out zygotes by three-dimensional imaging and IF semi-quantification.

Results: We find two distinct phases of loss of paternal methylation in the zygote, one prior to and another coincident with, but not dependent on, DNA replication. TET3-mediated hydroxymethylation is limited to the replication associated second phase of demethylation. Analysis of cytosine deaminase (AID) null fertilised oocytes revealed a role for this enzyme in the second phase of loss of paternal methylation, which is independent from hydroxymethylation. Investigation into the possible repair pathways involved supports a role for AID-mediated cytosine deamination with subsequent U-G mismatch long-patch BER by UNG2 while no evidence could be found for an involvement of TDG.

Conclusions: There are two observable phases of DNA demethylation in the mouse zygote, before and coincident with DNA replication. TET3 is only involved in the second phase of loss of methylation. Cytosine deamination and long-patch BER mediated by UNG2 appear to independently contribute to this second phase of active demethylation. Further work will be necessary to elucidate the mechanism(s) involved in the first phase of active demethylation that will potentially involve activities required for early sperm chromatin remodelling.
\end{abstract}

Keywords: Epigenetic reprogramming, DNA methylation, Hydroxymethylation, AID, BER, UNG2, TET3

\section{Background}

DNA methylation is an important epigenetic mark involved in gene silencing, $\mathrm{X}$ chromosome and transposon inactivation, genomic imprinting, and chromosome stability. DNA methylation is subject to reprogramming during development, involving both demethylation (active and passive) and de novo methylation phases. To date, the most clear examples of active DNA demethylation take place during the very early steps of mammalian development, namely in the zygote where the paternal genome undergoes a massive wave of loss of 5-methylcytosine (5mC) right after fertilisation [1-4].

\footnotetext{
* Correspondence: fatima.santos@babraham.ac.uk

'Epigenetics Programme, The Babraham Institute, Cambridge CB22 3AT, UK

Full list of author information is available at the end of the article
}

Within $1 \mathrm{~h}$ of fertilisation, the paternal genome goes through major chromatin remodelling, loses protamines and is re-packaged by maternal nucleosomal histones, forming the paternal pronucleus [5,6]. Post-fertilisation development can be defined by the pronuclear stages PN0/1 to PN5; PN0-PN2 embryos are in the G1 phase, PN3 and PN4 embryos are largely in S phase, replicating both the paternal and maternal genomes, and PN5 embryos are mostly in the post-replicative G2 phase [3,7-9]. Several reports have shown that the paternal genome undergoes genome-wide DNA demethylation via an active mechanism before replicating its DNA [1-4]. The search for enzymes responsible for this demethylation has produced numerous candidates and reaction mechanisms [10-13]. These fall within three main groups: (1) 
direct removal of the methyl group from the 5-C position of cytosine; (2) DNA repair, either base excision repair (BER) or nucleotide excision repair (NER); and (3) iterative enzymatic oxidation leading to conversion of $5 \mathrm{mC}$ to 5- hydroxymethylcytosine $(5 \mathrm{hmC}), 5$-formylcytosine $(5 \mathrm{fC})$, and 5-carboxylcytosine $(5 \mathrm{caC})$.

The methyl-CpG-binding domain protein 2 (MBD2) was reported to possess direct demethylase activity [14], but the result could not be reproduced by others. DNA glycosylases have been described in plants that can remove $5 \mathrm{mC}$, leaving an abasic site that is repaired by the BER machinery but mammalian glycosylases (e.g., thymine DNA glycosylase (TDG) and methyl-CpG-binding domain protein 4 (MBD4)) show weak activity on $5 \mathrm{mC}$ in vitro [15]. However, both MBD2 and MBD4 null fertilised oocytes undergo paternal loss of DNA methylation indistinguishable from matched controls [16].

An alternative to direct removal of $5 \mathrm{mC}$ by a DNA glycosylase is enzymatic deamination of $5 \mathrm{mC}$ to thymine, followed by T-G mismatch specific BER that replaces thymine with cytosine [17]. Two classes of enzymes have been proposed to be capable of carrying out the first step in this process: cytosine deaminases and DNA methyltransferases (reviewed in [11]). Cytosine DNA deaminases convert cytosine to uracil in nucleic acids and are well known from their roles in RNA editing, viral defence and antibody diversification [18]. Recently a series of results have pointed to an involvement of activation-induced deaminase (AID) mediated cytosine deamination in DNA demethylation in primordial germ cells (PGCs) and induced pluripotent stem (iPS) cell reprogramming, in cancer and embryonic stem (ES) cell gene expression [19-21]. Furthermore, overexpression of AID and MBD4 have been described to cause general demethylation of the zebrafish embryo genome, suggesting that deamination of $5 \mathrm{mC}$ followed by BER of T-G mismatches results in demethylation [22]. Gadd45, a p53-inducible gene involved in a variety of cellular processes, seems to facilitate this process and it has also been shown to interact with nucleotide excision repair (NER) components [22,23]. Models have been proposed for Gadd45 mediated demethylation of DNA either by deamination followed by BER or NER, or even by a combination involving consecutive NER and BER mechanisms (reviewed in [24]). AID's best defined activity is in B lymphocytes, where deamination of cytosines leading to uracil initiates both somatic hypermutation and immunoglobin class switch recombination [25-27]. However, its expression in mouse oocytes as well as in ES cells and PGCs [28], make it a potential candidate for performing global demethylation. In vitro assays have shown AID has $5 \mathrm{mC}$ deaminase activity, resulting in thymine and, therefore, T-G mismatches in DNA, which can be effectively repaired through the BER pathway [28].
Cytosine and 5-methylcytosine can also be enzymatically deaminated by DNA methyltransferases (DNMTs), primarily known as enzymes that transfer a methyl group to the C-5 position of cytosine from the methyl donor $\mathrm{S}$-adenosylmethionine (SAM- reviewed in [29]). Recent work in mammalian cell lines has led to the proposal that deamination by the Dnmt3a and Dnmt3b DNA methyltransferases could be a means of achieving fast, active DNA demethylation at promoters undergoing transcriptional cycling, by generating thymine, which is repaired via TDG and other enzymes [12,30-32]. More recently, studies have suggested that loss of DNA methylation in the paternal genome in the zygote is primarily dependent on TET3 [4,33], a member of the ten-eleven translocation (TET) family of DNA dioxygenases, which are capable of converting $5 \mathrm{mC}$ to 5 - hydroxymethylcytosine (5hmC), 5-formylcytosine ( $5 \mathrm{fC})$ and 5-carboxylcytosine $(5 \mathrm{caC})$ through iterative oxidation [34], suggesting that the main mechanism involved in active genome-wide demethylation is via oxidation of $5 \mathrm{mC}$. Although these various models for active loss of DNA methylation from the paternal pronucleus have been proposed, they have, for the most part, overlooked the evidence that this process occurs in two phases - before and coincident with DNA replication. To investigate this DNA methylation dynamics we have analysed fertilised mouse oocytes, by indirect immunofluorescence (IF) of DNA methylation and hydroxymethylation, and evaluated the relative contribution of different activities to active demethylation, by three-dimensional (3D) imaging and IF semi-quantification.

\section{Results and discussion}

We have previously characterised the dynamics of DNA methylation loss during the first cell cycle in the mouse by indirect immunofluorescence $[3,35]$. Since then, several similar studies have been published, more recently including other modifications of DNA methylation, namely hydroxymethylation, and attempts have been made to semi-quantify the immunofluorescence signals in order to get a sense of the kinetics of demethylation $[4,8,33,36,37]$. These approaches have provided clear evidence for the oxidation of $5 \mathrm{mC}$ in the zygote, specifically in the paternal pronucleus, seemingly explaining the concomitant decrease in DNA methylation.

We have established a protocol that allows us to reproducibly semi-quantify the staining levels of both DNA methylation and hydroxymethylation in pre-implantation embryos (see Methods and Additional files 1 and 2) and have used it to validate qualitative immunofluorescence results suggesting that, by the time the fertilised oocyte enters G1 a substantial amount of DNA methylation signal has already been lost from the paternal pronucleus, prior to the increase in the observed levels of 
hydroxymethylation (Figure 1A). To probe the kinetics of these two processes (loss of $5 \mathrm{mC}$ and increase in $5 \mathrm{hmC}$ ) we have captured $3 \mathrm{D}$ data of fertilised oocytes simultaneously stained for $5 \mathrm{mC}$ and $5 \mathrm{hmC}$. The signal intensity of both modifications was semi-quantified and the ratio between the paternal and maternal pronuclei in each individual one-cell embryo (from PN1 to PN5) calculated. The initial values for the two complements cannot be measured using this protocol as condensed sperm is notably impervious to immunostaining due to its highly compacted chromatin state [6], resulting in unreliable semi-quantification values for PN0. Based on the levels of DNA methylation for mouse sperm and oocytes, reported by genome-wide or reduced representation bisulphite-sequencing, and despite variability in the quantification methods, there is a general agreement that DNA methylation levels in sperm are twice that observed in the oocyte $[38,39]$. It is therefore possible to calculate a conservative (using the lowest estimate based on whole-genome bisulphite-sequencing, [38]) theoretical paternal to maternal ratio of DNA methylation of 2 for PNO (Figure 1B). Functional grouping of the data according to cell cycle [8], corresponding to embryos in G1 (Early; PN1-PN2) and in S-G2 phase (Mid-Late; PN3-PN5), was used to compare our data with that collected by others. A summary of paternal to maternal ratios

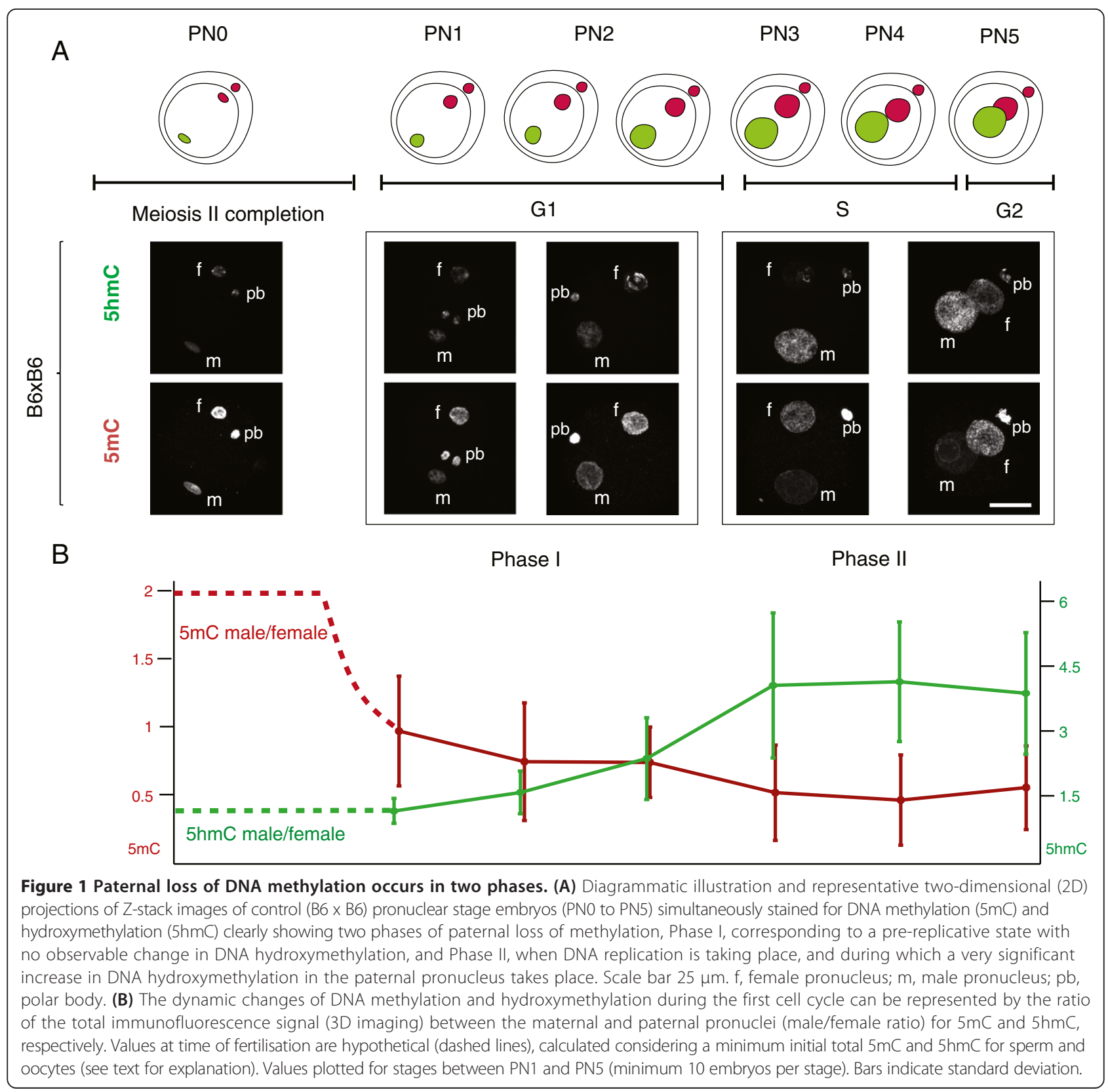


of $5 \mathrm{mC}$ levels based on reports from the literature, as well as this work, is presented in Table 1. From its examination it becomes clear that, in all cases and consistently throughout the data generated by multiple independent labs, the paternal/maternal ratio of DNA methylation in G1 is never close to the theoretical initial PN0 value of 2, predicted from the $5 \mathrm{mC}$ levels reported for the gametes. There is thus an observable first loss of paternal DNA methylation levels of approximately $60 \%$ by the time the zygote enters G1 (average ratio drop from 2 to 0.8 ) and a further loss of $20 \%$ (average ratio drop from 0.8 to 0.5 ) thereafter. This supports the distinction between two phases of active demethylation, one in G1 and the other in S-G2 (Figure 1A, B). In contrast, the levels of hydroxymethylation, evaluated the same way, show a rather different profile. While the paternal/maternal ratio remains relatively stable during Phase $\mathrm{I}$, there is a remarkable increase during Phase II with a marked rise in the $5 \mathrm{hmC}$ signal in the paternal pronucleus (Figure 1A, B). Although concomitant with S-phase, this increase in paternal hydroxymethylation is not dependent on DNA replication ([4] and Additional file 3). This is again in agreement with our analysis of results reported in the literature, which shows a significant increase in the $5 \mathrm{hmC}$ paternal/maternal ratio occurring between Early (Phase I) and Mid-Late (Phase II) one-cell embryos (Table 2). The changes in $5 \mathrm{hmC}$ signal point to the involvement of TET-mediated $5 \mathrm{mC}$ oxidation during Phase II, but not Phase I, of paternal loss of DNA methylation. In order to test this hypothesis, we used a conditional (Zp3-Cre) TET3 knock-out mouse generated in the lab. TET3 null oocytes were fertilised by $\mathrm{C} 57 \mathrm{Bl} / 6 \mathrm{~J}$ (B6) sperm and the same simultaneous $5 \mathrm{mC}$ and $5 \mathrm{hmC}$ staining and semi-quantification analysis was performed (Figure 2). The results confirmed the dependency of paternal hydroxymethylation on the maternally derived TET3 protein [33] with no observable increase in $5 \mathrm{hmC}$ staining in Mid-Late one-cell embryos (Figure 2A) and consequently a very significant difference in the paternal/maternal ratios between TET3 MAT KO and control B6 fertilised oocytes (Figure 2B). The absence of paternal $5 \mathrm{hmC}$ signal increase was accompanied by an equally significant rise in paternal DNA methylation staining levels in PN3-PN5 one-cell

Table 1 DNA methylation $(5 \mathrm{mC})$ average paternal to maternal ratios (indirect immunofluorescence)

\begin{tabular}{llll}
\hline Study & $\begin{array}{l}\text { Early } \\
\text { (PN1-PN2) }\end{array}$ & $\begin{array}{l}\text { Mid-Late } \\
\text { (PN3-PN5) }\end{array}$ & Mouse strain \\
\hline lqbal et al., 2011 $^{1}$ & 0.9 & 0.47 & FVB \\
Wossidlo et al., 2011 $^{1}$ & 0.53 & 0.34 & BDF1 (C57BL/6 x DBA) \\
Inoue and Zhang, 2011 & 0.85 & 0.61 & BDF1 (C57BL/6 x DBA) \\
Salvaing et al., 2012 & 0.72 & 0.47 & F1 (C57BL/6 x CBA) \\
This study & 0.84 & 0.62 & C57BL/6 J \\
\hline
\end{tabular}

${ }^{1}$ DNA staining was used for normalisation of paternal/maternal ratios.
Table 2 DNA hydroxymethylation ( $5 \mathrm{hmC}$ ) average paternal to maternal ratios (indirect immunofluorescence)

\begin{tabular}{llll}
\hline Study & $\begin{array}{l}\text { Early } \\
\text { (PN1-PN2) }\end{array}$ & $\begin{array}{l}\text { Mid-Late } \\
\text { (PN3-PN5) }\end{array}$ & Mouse strain \\
\hline lqbal et al., 2011 & 0.78 & 2.82 & $\mathrm{FVB}$ \\
Wossidlo et al., 2011 $^{1}$ & 0.85 & 2.44 & $\mathrm{BDF1}(\mathrm{C} 57 \mathrm{BL} / 6 \times \mathrm{DBA})$ \\
Inoue and Zhang, 2011 $^{1}$ & 1.89 & 4.98 & $\mathrm{BDF} 1(\mathrm{C} 57 \mathrm{BL} / 6 \times \mathrm{DBA})$ \\
Salvaing et al., 2012 & 0.9 & 2.23 & $\mathrm{~F} 1(\mathrm{C} 57 \mathrm{BL} / 6 \times \mathrm{CBA})$ \\
This study & 1.334 & 4.01 & $\mathrm{C57BL} / 6 \mathrm{~J}$ \\
\hline
\end{tabular}

${ }^{1}$ DNA staining was used for normalisation of paternal/maternal ratios.

embryos (Figure 2B), supporting a role for TET3-mediated hydroxymethylation in the loss of paternal DNA methylation at this stage. We then concentrated our attention on the first phase of paternal DNA methylation loss that seems to be independent of hydroxymethylation. DNA repair has been proposed as a mechanism to explain active DNA demethylation and there is evidence for DNA repair pathways being involved in paternal DNA methylation loss in the zygote, particularly BER $[11,40,41]$. Moreover, deamination has been implicated as a possible upstream event initiating the BER mediated demethylation [42]. Activation-induced cytidine deaminase has also been shown to be capable of deaminating $5 \mathrm{mC}$ and is expressed in pluripotent tissues, including mouse oocytes [28]. AID was initially thought to be only relevant in B-lymphocytes, where it is essential for somatic hypermutation and class-switch recombination [26,27]. Recently AID has been shown to be involved in dynamic methylation changes in a variety of tissues ranging from PGCs [21] to ES and iPS cells $[20,43]$. The presence of AID protein in control mouse oocytes was confirmed by indirect immunofluorescence (Additional file 4). Making use of the same AID knock-out mouse model that showed altered DNA methylation levels in PGCs [21], AID null oocytes were fertilised by either AID (Additional file 5) or B6 (Figure 3) sperm and the same simultaneous $5 \mathrm{mC}$ and $5 \mathrm{hmC}$ staining and semi-quantification analysis was performed. A significant difference was found in the paternal/maternal ratio of DNA methylation between AID and control (B6) fertilised oocytes, with no apparent effect on the levels of hydroxymethylation (Figure 3B). Notably, this difference was only detectable in PN3-PN5 (Phase II) embryos and no difference could be seen in PN1-PN2 (Phase I) zygotes (Figure 3A and Additional file 5) despite reports indicating that AID works during G1 [44]. AID deaminates cytosines at immunoglobulin genes on single-stranded DNA thought to be made available during transcription [45], however post-fertilisation mouse oocytes are transcriptionally silent [46] thus necessitating other means of generating singlestranded DNA substrates. As such, it is reasonable to expect that AID might be able to deaminate immediately 


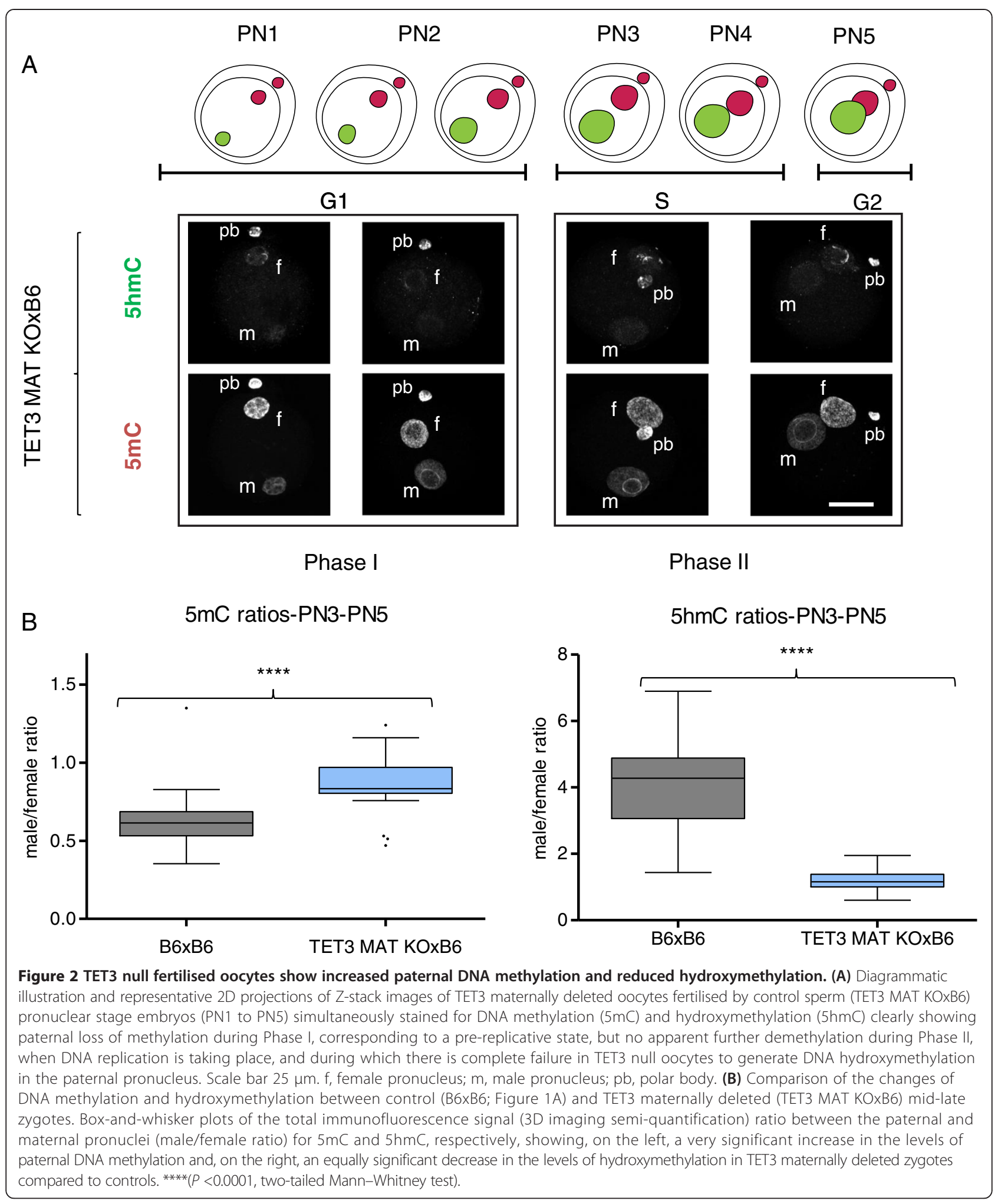

after fertilisation during nucleoprotamine exchange, a time when single-stranded DNA would be accessible. Recently it has been described that, in both class-switching B-cells and E. coli, negative DNA supercoiling, and hence generation of single-stranded DNA, enhances AID mutagenesis [47]. Within $1 \mathrm{~h}$ after fertilisation the paternal chromatin suffers a complete remodelling resulting in an exchange of the sperm protamines by maternally derived 


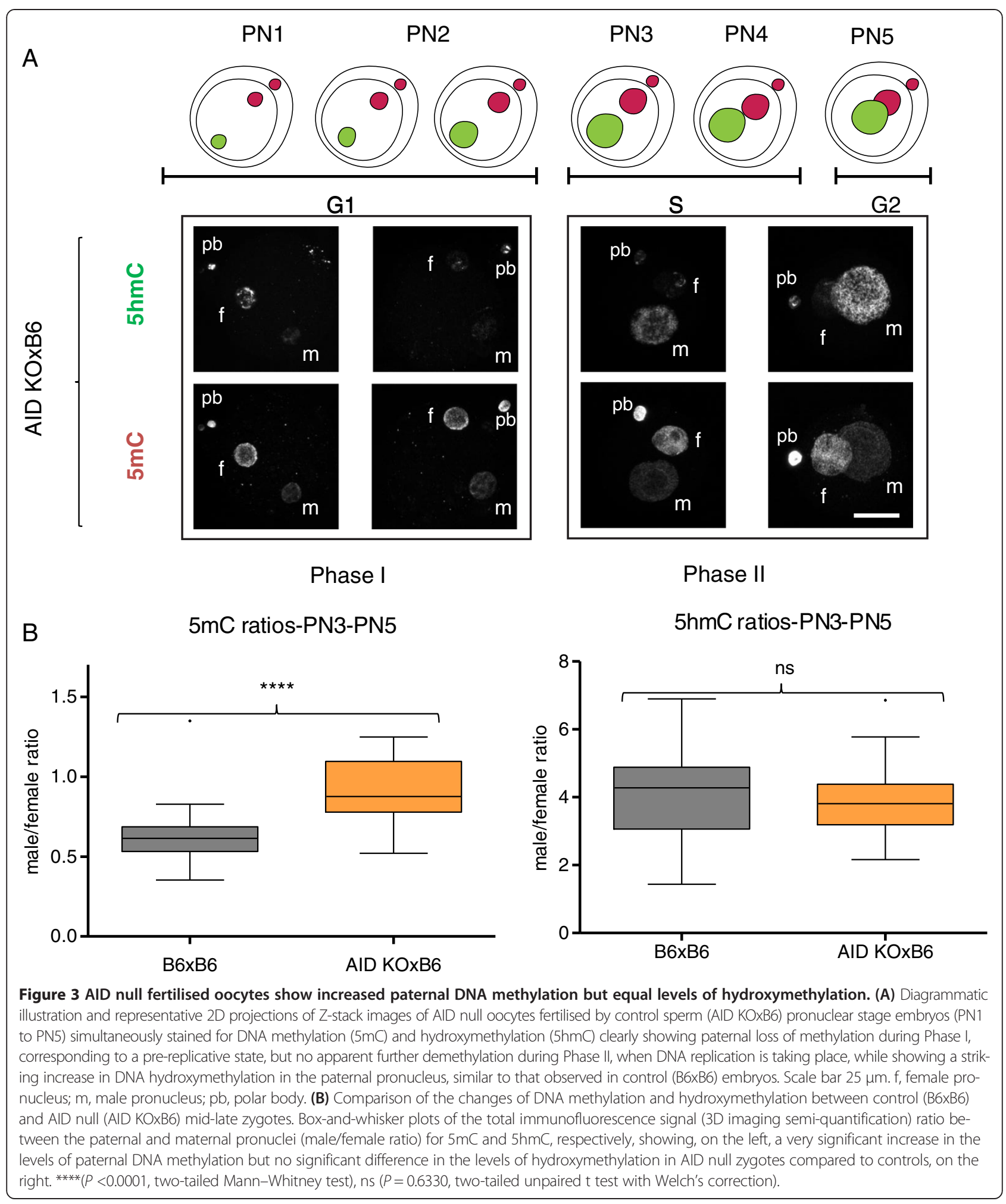

histones $[5,6]$. This process will likely involve the generation of DNA supercoils, mirroring what happens in the course of nucleosome elimination and incorporation of protamines during spermiogenesis [48], which may facilitate AID mediated deamination. Deamination of $5 \mathrm{mC}$ during $\mathrm{G} 1$ in the fertilised oocyte would immediately result in loss of DNA methylation immunofluorescence signal, given the high specificity of the antibody used, leading to an expected increase of the paternal/maternal $5 \mathrm{mC}$ ratio in PN1-PN2 (G1) staged 
zygotes compared to B6 controls. The fact that no significant difference could be found suggests that no such deamination has occurred in the control zygotes. The results obtained were therefore surprising. Investigating the known mechanisms for DNA repair following AIDmediated deamination provided a possible explanation (Additional file 6). Repair can be achieved by different mechanisms, both error-prone and error-free [49]. Deamination of $5 \mathrm{mC}$ generates $\mathrm{T}$ creating a $\mathrm{T}-\mathrm{G}$ mismatch which is the preferred substrate for TDG and MBD4 glycosylases $[28,50]$. On the other hand, deamination of $C$ generates $U$ and uracil residues in DNA are also largely resolved by BER, with the uracil being removed by a DNA glycosylase [50]. Uracyl glycosylase (UNG2) has been implicated as the major glycosylase responsible for repair of $\mathrm{C}$ to $\mathrm{U}$ mismatches following deamination [51]. BER enables the repair of damaged DNA via two general pathways, short-patch and long-patch [50,52,53]. Short-patch (SP) BER replaces a single nucleotide by polymerase $\beta$ and the newly synthesized DNA is sealed by DNA ligase III/Xray cross-complementing group 1 (XRCC1) heterodimer. Long-patch (LP) BER inserts two to 13 nucleotides by coordinate action of polymerase $\delta$, proliferating cell nuclear antigen (PCNA), flap endonuclease 1 and DNA ligase I. Although at face value only the deamination of $5 \mathrm{mC}$ could result in demethylation, in the case of cytosine deamination followed by LP BER, there would be the possibility for any methylated cytosines adjacent to the U-G mismatch to be replaced by newly incorporated non-modified cytosines and the original methylated state would be lost, resulting in demethylation (Additional file 6). It is thus possible to achieve DNA methylation loss through a $C$ to $\mathrm{U}$ deamination and subsequent replacement of adjacent methylated cytosines by LP BER (Figure 4A). To resolve these potential alternative downstream pathways, analysis of knock-out mouse oocytes for either TDG [54] or UNG2 [55], fertilised by B6 sperm, was performed (Figure 4B). The results support a role for AID mediated cytosine deamination with subsequent U-G mismatch LP BER and no evidence could be found for direct $5 \mathrm{mC}$ deamination and T-G mismatch repair. This is in agreement with the reported reduced activity of AID on $5 \mathrm{mC}$ when compared to cytosine, its canonical substrate $[10,28]$. Furthermore, PARP1, a hallmark of LP BER [52,53] (Additional file 6), has been found to be predominantly confined to the paternal pronucleus in PN3-PN5 staged zygotes $[40,41]$ and its specific inhibition caused a significant increase in the paternal/maternal ratio of DNA methylation compared to controls [40]. In this scenario, AID-mediated cytosine deamination can still occur in G1 (PN1-PN2), without causing any immediate $5 \mathrm{mC}$ loss, and the resulting U-G mismatches could later be repaired by LP BER, presumably causing loss of adjacent methylated cytosines with consequent demethylation in PN3-
PN5 staged zygotes. Although speculative, this model can fully account for the results obtained.

According to recent reports, deamination of cytosine and 5-methylcytosine could also be mediated by DNA methyltransferases, namely the Dnmt3a and Dnmt3b de novo methyltransferases (reviewed in [11,12]) and more recently that DNA could be directly demethylated by the same enzymes, at least in an in vitro system [56]. Moreover, results from ES cells suggest that TET and Dnmt3 enzymes may interact at common binding sites [57]. These prompted us to investigate whether Dnmt3a null oocytes were capable of demethylating a control (B6) sperm using a conditional (Zp3-Cre) Dnmt3a knock-out mouse model [58]. Oocytes null for Dnmt3a are severely depleted of DNA methylation [59] which precluded the use of the paternal/maternal ratio as a measure of demethylation as the maternal pronucleus showed only residual levels of $5 \mathrm{mC}$ staining (Additional file 7A); instead we directly compared the total levels of immunofluorescence signal in the paternal pronuclei of Dnmt3a null, control (B6) and AID null oocytes fertilised by B6 sperm (Additional file 7B). No significant difference, in either $5 \mathrm{mC}$ or $5 \mathrm{hmC}$ levels, between paternal pronuclei in control and Dnmt3a null fertilised oocytes was observed, whereas both are significantly different from AID null in $5 \mathrm{mC}$ but not in $5 \mathrm{hmC}$ levels (Additional file $7 \mathrm{~B}$ ). These results do not support a role for Dnmt3a in paternal active demethylation in the mouse one-cell embryo.

As a whole, our results support a role for both $5 \mathrm{mC}$ hydroxymethylation and cytosine deamination, followed by LP BER, as demethylation mechanisms in the mouse zygote. Both these mechanisms seem to contribute independently to a decrease in paternal DNA methylation coincident, but not reliant, on DNA replication. Furthermore, an initial loss of paternal DNA methylation, prior to S-phase, seems to take place, for which none of the activities investigated in this work seem to supply an explanation. It is likely that this early demethylation is related to the need for major chromatin remodelling of the sperm within the first hours post fertilisation and may involve other pathways of DNA repair. NER has been suggested as a candidate for DNA demethylation (reviewed in $[10,11]$ ), removes bulky DNA lesions and is a multistep process involving the action of as many as 20 to 30 proteins working in a well-defined sequence [60]. Other repair pathways, such as non-homologous end joining (NHEJ) and homologous recombination (HR), could also play a role in this first phase of paternal loss of methylation [61]. The complexity, and possible redundancy, of these alternative pathways will require considerable research effort in order to elucidate this early DNA methylation loss. 


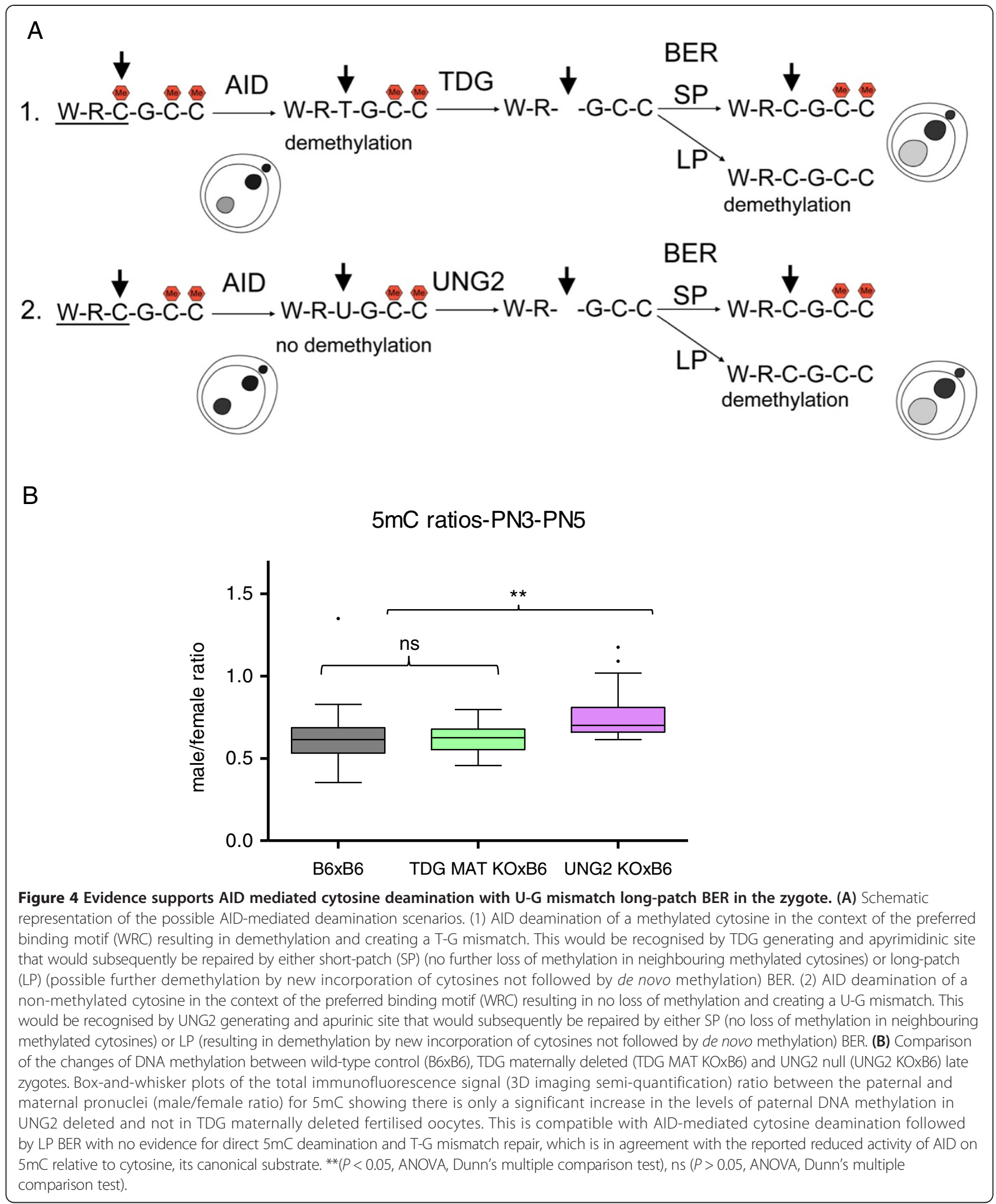

\section{Conclusions}

Our analysis shows that there are two observable phases of active DNA demethylation in the mouse zygote, before and coincident with DNA replication. We further show that TET3 seems to be involved only in the second phase of loss of methylation. Cytosine deamination and uracil BER seem to also independently contribute to this second phase of active demethylation. Together these 
findings allow us to conclude that demethylation is achieved by at least two parallel mechanisms which may or may not be partially redundant. Our results highlight the dynamic nature of DNA demethylation, with two apparent distinct stages (Phase I and Phase II). The major increase in $5 \mathrm{hmC}$ (Phase II) seems to be uncoupled from the initial loss of DNA methylation (Phase I) which was not previously acknowledged. Still, further work will be necessary to elucidate the mechanism(s) involved in the first phase of demethylation, likely to involve activities required for early chromatin remodelling on fertilisation and perhaps other types of DNA repair.

To our knowledge, this is the first report of possible involvement of LP BER in DNA demethylation, opening new avenues of investigation not formerly considered.

\section{Methods}

Mice and sample collection

All experimental procedures were approved by the Animal Welfare, Experimentation and Ethics Committee (AWEEC) at the Babraham Institute and were performed under licenses by the Home Office (UK) in accordance with the Animals (Scientific Procedures) Act 1986.

Fertilised mouse oocytes were collected on the day of plugging from naturally mated inbred C57BL/6 J (B6) mice supplied from breeding colonies in the Biological Support Unit at the Babraham Institute. Mechanistic investigation into the loss of DNA methylation was made possible through the generation of conditional deletion of key activities, Dnmt3a [62] and TDG [54] (rescued by a floxed TDG minigene), derived by breeding female mice homozygous for floxed alleles together with the Zp3 Cre recombinase transgene [63]. Conditionally deleted oocytes generated in this way were referred to as maternal knock-outs (MAT KO). In all cases these MAT $\mathrm{KO}$ generating females were bred to wild-type B6 control males. Constitutively deleted activities were derived for AID [26] and UNG2 [51,55] from homozygous females null for the respective enzymes.

Individual zygote pronuclear staging was performed as previously described [3]. The cell-cycle state of these different stages has been characterised according to the literature, establishing that zygotes between PN1 and PN2 will be in G1 and from PN3 to PN5 in S-G2 [8].

\section{Generation of TET3 conditional deletion}

C57BL/6 N Tac ES cells (TaconicArtemis) were targeted with a vector introducing LoxP sites around exon 5 of Tet3 RefSeq NM_183138.2 (sequence: CCGGACCTGTG CTTGCCAAGGCAAAGACCCTAACACCTGCGGTGC CTCCTTCTCCTTCGGCTGTTCCTGGAGCATGTACT TCAACGGCTGCAAATATGCTCGGAGCAAGACGCCA
CGAAAGTTCCGCCTCACGGGAGACAATCCGAAGG AG) which encodes residues required for chelation of $\mathrm{Fe}(\mathrm{II})$ and is upstream of exons containing other key catalytic residues [64]. Expression of Cre recombinase results in excision of this region and a frame-shift from exon 6 affecting all downstream exons until a premature stop codon in exon 7. Animals heterozygous for the floxed allele were bred to a transgenic mouse line containing Zp3 Cre on a B6 background [63] and homozygous mice were generated by inter-crossing to give females of the appropriate genotype.

\section{Immunofluorescence and confocal microscopy}

Antibody staining of DNA methylation (Eurogentec, BIMECY) and hydroxymethylation (Active Motif, 39769) was performed as previously described [9] with modifications. Briefly, zygotes were fixed with 4\% PFA for 15 min and, after permeabilisation with $0.5 \%$ Triton X-100, the samples were treated with $4 \mathrm{~N} \mathrm{HCl}$ for $10 \mathrm{~min}$ at room temperature, washed in PBS/Tween and blocked overnight; simultaneous incubation with both primary antibodies followed by simultaneous secondary detection (AlexaFluor, Molecular probes, Invitrogen) was used. To allow for full 3D sample capture the samples were mounted in fibrin clots [65]. Image acquisition was performed with a LSM 510 Meta confocal laser scanning microscope (Carl Zeiss) equipped with a 'PlanApochromat' 63x/1.40 DIC oil-immersion objective and an Olympus FV1000 equipped with a UPLSAPO 60x/1.35 DIC oil-immersion objective. DNA was counterstained with YOYO1 ${ }^{\mathrm{mm}}$ (Molecular Probes, Life Technologies). Zstacks of 20 to 65 optical sections were collected from each zygote (700x700, pixel size; z-step, $0.50 \mu \mathrm{m})$. At least 10 zygotes of each group were imaged from at least two biological replicates. Images were pseudo-coloured using Adobe Photoshop CS4.

\section{Semi-quantification and statistics}

Fluorescence semi-quantification analysis (total sum, 3D rendering) was performed as follows, 3D reconstruction of confocal image stacks was performed using Volocity 5.5 (Improvision), after which regions of interest (ROIs) were defined around each pronucleus and total voxel count signal intensity (SUM) for each channel was computed. Examples of each of these steps can be found in Additional file 1. The data were then exported into Excel and the individual maternal to paternal ratios (male/ female ratios) for each zygote calculated as this measure is widely used in the field and allows for a good degree of comparison between studies. Statistical analysis (analysis of variance (ANOVA), Mann-Whitney or unpaired $t$ test) and whisker-plot graphs were performed with GraphPad Prism 5 and 6. 


\section{Additional files}

Additional file 1: 3D reconstruction of confocal image stacks and total fluorescence semi-quantification. Volocity 5.5 (Improvision) was used for 3D rendering and signal semi-quantification of each individual embryo Z-stack. (A) Screen-shot showing an XYZ view of a representative Z-stack. (B) Screen-shot showing the 3D rendering of the same Z-stack. (C) Screen-shot showing the regions of interest (ROls) defined around each of the objects inside the sample, paternal pronucleus (red), maternal pronucleus (green) and polar body (blue). (D) Screen-shot showing the protocol to define the ROls and subsequent computation of several measurements, including Sum signal intensity for each of the channels, used as a measure of the total signal for $5 \mathrm{hmC}$ (Channel: 2 ) and $5 \mathrm{mC}$ (Channel: 3).

Additional file 2: Fluorescence semi-quantification protocol validation data. (A) Four independent samples of B6xB6 generated fertilised oocytes between PN3 and PN5 were evaluated using the optimized protocol for simultaneous staining of $5 \mathrm{mC}$ and $5 \mathrm{hmC}, 3 \mathrm{D}$ image acquisition and semi-quantification. Statistical analysis (ANOVA) shows no significant differences can be found between the four replicates. (B) Mouse embryonic stem cells (E14) were cultured in both serum and 2i conditions [57] and analysed for global DNA methylation levels by using the optimised immunofluorescence semi-quantification protocol (left) or by mass-spectrometry (right). Both methods are in agreement both qualitatively (E14 serum > E14 2i) and quantitatively (E14 serum 40\% to $50 \%$ more methylated than E14 2i).

Additional file 3: Replication inhibition does not affect DNA methylation or hydroxymethylation paternal/maternal ratios in the zygote. Two independent replicates of at least 15 B6xB6 early fertilised oocytes were collected and incubated in M16 medium (M-7292-SIGMA) supplemented with either $2.5 \mathrm{\mu L} / \mathrm{mL}$ DMSO (control) or $2.5 \mu \mathrm{L} / \mathrm{mL}$ Aphidicolin (A4487-SIGMA) and cultured $\left(37^{\circ} \mathrm{C} ; 5 \% \mathrm{CO}_{2}\right)$ for $5 \mathrm{~h}$. For replication analysis both groups (control-DMSO and Aphidicolin) were then transferred to a fresh same composition medium drop, to which 20 $\mu \mathrm{M}$ EdU (Click-iT TM EdU Alexa Fluor ${ }^{\circledR}$ 488, Invitrogen) was added, for a further $1 \mathrm{~h}$ (detection according to the manufacturer's instructions). (A) Representative images of control (DMSO) and replication inhibited (Aphidicolin) embryos. Single optical slices. EdU-green; 5 hmC-red; $5 \mathrm{mC}$-white. Scale bar $25 \mu \mathrm{m}$. f, female pronucleus; $\mathrm{m}$, male pronucleus; pb, polar body. (B) Comparison of the changes of DNA methylation and hydroxymethylation between control (DMSO) and replication inhibited (Aphidicolin) mid-late zygotes. Box-and-whisker plots of the total immunofluorescence signal (3D imaging semiquantification) ratio between the paternal and maternal pronuclei (male/female ratio) for $5 \mathrm{mC}$ and $5 \mathrm{hmC}$, respectively, showing no significant difference (unpaired t test) in the levels of methylation (left, $P=0.7842$ ) or hydroxymethylation (right, $P=0.0748$ ) in Aphidicolin-treated zygotes compared to controls (DMSO).

Additional file 4: AID is expressed in mouse oocytes and localises to the pronuclei. Wild-type controls (B6XB6) and AID null (AID KO $x$ (C57BI/6JXCBA)-F1) zygotes were stained with an antibody against AID (A15, Santa Cruz Biotechnology) and DAPI. The control zygotes show a typical predominantly cytoplasmic localisation of AID protein, as has been described for B-cells (reviewed in [66]), but there is visible signal in both pronuclei that is completely absent in the AID null fertilised oocytes. Scale bar 25 mm. f, female pronucleus; m, male pronucleus; pb, polar body.

Additional file 5: AID null zygotes show no significant $5 \mathrm{mC}$ difference during Phase I of paternal demethylation. Comparison of the changes of DNA methylation and hydroxymethylation between wild-type control (B6xB6) and AID null (AIDxAID) Phase I (PN1-PN2) and Phase II (PN3-PN5) zygotes. Box-and-whisker plots of the total immunofluorescence signal (3D imaging semi-quantification) ratio between the paternal and maternal pronuclei (male/female ratio) for $5 \mathrm{mC}$ and $5 \mathrm{hmC}$, respectively, showing, on the left, a very significant increase in the levels of paternal DNA methylation in Phase II but no significant difference in Phase I fertilised oocytes. No significant differences could be found in the levels of hydroxymethylation in AID null compared to wild-type in either Phase I or Phase II zygotes (on the right). ${ }^{* * *}(P<0.0001$, Mann-Whitney test), ns ( $P>0.05$, Unpaired t test).
Additional file 6: AID-mediated deamination and BER SP and LP pathways. Diagram of the SP and LP BER pathways for AID-mediated DNA deamination. AID has been shown in vitro and in E. coli to be capable of deaminating 5-methylcytosine ( $5 \mathrm{mC})$, generating T-G mismatches and thus directly removing methylation from DNA (left hand-side), although the preferred substrate is cytosine, very efficiently generating $\mathrm{U}-\mathrm{G}$ mismatches but having no direct effect on DNA methylation loss (right hand-side). After removal of the mismatched base (black circles) by a DNA glycosylase (TDG in the case of a T-G mismatch and UNG2 in the case of an U-G mismatch) and incision by apurinic/apyrimidinic endonuclease (APE1), BER may proceed by the SP repair or by the LP repair. SP BER replaces a single nucleotide by polymerase $\beta$ and the newly synthesized DNA sealed by DNA ligase III/X-ray cross-complementing group 1 (XRCC1) heterodimer. LP BER inserts two to 13 nucleotides by concordant action of polymerase $\delta$, proliferating cell nuclear antigen (PCNA), flap endonuclease 1 and DNA ligase I. In this case, any methylated cytosines adjacent to the generated U-G mismatch would be replaced by new cytosines and, if not subsequently de novo methylated, the original methylated state would be lost resulting in demethylation. Poly ADP Ribose Polymerase 1 (PARP1), which binds to and is activated by DNA strand breaks, has been implicated in LP repair promoting the rapid recruitment of PAR-binding proteins to the site of DNA damage, which is important for efficient damage repair (modified from [52]).

Additional file 7: No evidence for Dnmt3a mediated de novo DNA methylation in the paternal pronucleus. (A) Representative $2 D$ projections of Z-stack images of control (B6xB6) and Dmnt3a maternally deleted oocytes fertilised by control sperm (Dnmt3a MAT KOxB6) late pronuclear stage embryos (PN3) simultaneously stained for DNA methylation (5mC- red) and hydroxymethylation (5hmC-green) showing no difference in both paternal loss of methylation and acquisition of hydroxymethylation, but a very obvious lack of maternal DNA methylation. Inset, merge with DNA staining (YOYO1) - blue. Scale bar $25 \mu \mathrm{m}$. f, female pronucleus; $m$, male pronucleus; pb, polar body. (B) Comparison of the changes of DNA methylation and hydroxymethylation between control (B6xB6), Dmnt3a maternally deleted (Dnmt3a MAT KOxB6) and AID null (AIDxB6) mid-late (PN3-PN5) zygotes. Box-and-whisker plots of the total paternal (male) pronucleus indirect immunofluorescence signal (3D imaging semi-quantification) for $5 \mathrm{mC}$ and $5 \mathrm{hmC}$ show no significant difference in either the levels of paternal DNA methylation $(5 \mathrm{mC})$ in Dnmt3a MAT KO fertilised oocytes relative to controls, on the left, or of hydroxymethylation $(5 \mathrm{hmC})$, on the right. ${ }^{* * * *}(P<0.0001$, ANOVA); ns $(P>0.05$, ANOVA)

\section{Abbreviations}

2D: Two-dimensional; 3D: Three-dimensional; 5caC: 5-carboxylcytosine; 5fC: 5-formylcytosine; $5 \mathrm{hmC}$ : 5-hydroxymethylcytosine; $5 \mathrm{mC}$ : 5-methylcytosine; AID: Activation-induced deaminase; ANOVA: Analysis of variance; APE1: Apurinic-apyrimidinic endonuclease 1; B6: C57BL/6 J mouse strain BER: Base excision repair; C: Cytosine; DNA: Deoxyribonucleic acid; ES cells: Embryonic stem cells; Dnmt3a: DNA (cytosine-5)-methyltransferase 3a; Dnmt3b: DNA (cytosine-5)-methyltransferase 3b; G: Guanosine; HCl: Hydrochloric acid; HR: Homologous recombination; IF: Immunofluorescence; iPS cells: induced pluripotent stem cells; LP: Longpatch; MAT KO: Maternal knock-out; MBD2: Methyl-CpG binding Domain 2; MBD4: Methyl-CpG binding Domain 4; NER: Nucleotide excision repair; NHEJ: Non-homologous end joining; PARP1: poly-ADP-ribose polymerase 1; PCNA: Proliferating cell nuclear antigen; PGCs: Primordial germ cells; ROls: Regions of interest; SP: Short-patch; T: Thymine; TDG: Thymine DNA glycosylase; TET3: Ten-eleven translocation 3; U: Uracil; UNG2: Uracil-DNA glycosylase 2; XRCC1: X-ray cross-complementing group 1.

\section{Competing interests}

The authors declare that they have no competing interests.

\section{Authors' contributions}

FS, WD and WR generated the main idea of the work and developed the study design, both conceptually and methodologically. WD organised and collected the samples. FS acquired and analysed the data. FS, WD and WR contributed to analysis and interpretation of the data. CR contributed materials. JP and HB were responsible for mouse genotyping of TET3 and 
TDG strains, respectively. FS, WD and WR wrote the manuscript. FS, WD, JP, $H B, C R$ and WR made comments, suggested appropriate modifications and made corrections. All authors read and approved the final manuscript.

\section{Authors' information}

Wolf Reik and Wendy Dean are senior authors.

\section{Acknowledgements}

This work was supported by funding from the Biotechnology and Biological Sciences Research Council (BBSRC), the Medical Research Council (MRC) and The Wellcome Trust. The authors would like to thank Dr Hiro Sasaki for providing the Dnmt3a mice used in this study, Dr Primo Schär for the TDG materials and Dr Gabriella Ficz for the initial design of the TET3 knock-out.

\section{Author details}

${ }^{1}$ Epigenetics Programme, The Babraham Institute, Cambridge CB22 3AT, UK. ${ }^{2}$ Laboratory of Molecular Biology, Francis Crick Avenue, Cambridge CB2 0QH, UK. ${ }^{3}$ Centre for Trophoblast Research, University of Cambridge, Cambridge CB2 3EG, UK

Received: 22 August 2013 Accepted: 30 October 2013

Published: 14 November 2013

\section{References}

1. Mayer W, Niveleau A, Walter J, Fundele R, Haaf T: Demethylation of the zygotic paternal genome. Nature 2000, 403:501-502.

2. Oswald J, Engemann S, Lane N, Mayer W, Olek A, Fundele R, Dean W, Reik W, Walter J: Active demethylation of the paternal genome in the mouse zygote. Curr Biol 2000, 10:475-478.

3. Santos F, Hendrich B, Reik W, Dean W: Dynamic reprogramming of DNA methylation in the early mouse embryo. Dev Biol 2002, 241:172-182.

4. Wossidlo M, Nakamura T, Lepikhov K, Marques CJ, Zakhartchenko V, Boiani M, Arand J, Nakano T, Reik W, Walter J: 5-Hydroxymethylcytosine in the mammalian zygote is linked with epigenetic reprogramming. Nat Commun 2011, 2:241.

5. McLay DW, Clarke HJ: Remodelling the paternal chromatin at fertilization in mammals. Reproduction 2003, 125:625-633.

6. van der Heijden GW, Dieker JW, Derijck AA, Muller S, Berden JH, Braat DD, van der Vlag J, de Boer P: Asymmetry in histone H3 variants and lysine methylation between paternal and maternal chromatin of the early mouse zygote. Mech Dev 2005, 122:1008-1022.

7. Adenot PG, Mercier Y, Renard JP, Thompson EM: Differential H4 acetylation of paternal and maternal chromatin precedes DNA replication and differential transcriptional activity in pronuclei of 1-cell mouse embryos. Development 1997, 124:4615-4625.

8. Salvaing J, Aguirre-Lavin T, Boulesteix C, Lehmann G, Debey P, Beaujean N: 5-Methylcytosine and 5-hydroxymethylcytosine spatiotemporal profiles in the mouse zygote. PloS one 2012, 7:e38156.

9. Santos F, Peters AH, Otte AP, Reik W, Dean W: Dynamic chromatin modifications characterise the first cell cycle in mouse embryos. Dev Biol 2005, 280:225-236

10. Franchini DM, Schmitz KM, Petersen-Mahrt SK: 5-Methylcytosine DNA demethylation: more than losing a methyl group. Annu Rev Genet 2012, 46:419-441.

11. Gehring M, Reik W, Henikoff S: DNA demethylation by DNA repair. Trends Genet 2009, 25:82-90.

12. Ooi SK, Bestor TH: The colorful history of active DNA demethylation Cell 2008, 133:1145-1148.

13. Wu SC, Zhang Y: Active DNA demethylation: many roads lead to Rome. Nat Rev Mol Cell Biol 2010, 11:607-620.

14. Bhattacharya SK, Ramchandani S, Cervoni N, Szyf M: A mammalian protein with specific demethylase activity for mCpG DNA. Nature 1999, 397:579-583.

15. Zhu JK: Active DNA demethylation mediated by DNA glycosylases. Annu Rev Genet 2009, 43:143-166.

16. Santos F, Dean W: Epigenetic reprogramming during early developmen in mammals. Reproduction 2004, 127:643-651.

17. Wiebauer K, Jiricny J: Mismatch-specific thymine DNA glycosylase and DNA polymerase beta mediate the correction of G.T mispairs in nuclear extracts from human cells. Proc Natl Acad Sci U S A 1990, 87:5842-5845.

18. Conticello SG: The AID/APOBEC family of nucleic acid mutators. Genome Biol 2008, 9:229.
19. Isobe T, Song SN, Tiwari $P$, Ito H, Yamaguchi Y, Yoshizaki K: Activationinduced cytidine deaminase auto-activates and triggers aberrant gene expression. FEBS Lett 2013, 587:2487-2492.

20. Kumar R, DiMenna L, Schrode N, Liu TC, Franck P, Munoz-Descalzo S, Hadjantonakis AK, Zarrin AA, Chaudhuri J, Elemento O, Evans T: AID stabilizes stem-cell phenotype by removing epigenetic memory of pluripotency genes. Nature 2013, 500:89-92.

21. Popp C, Dean W, Feng S, Cokus SJ, Andrews S, Pellegrini M, Jacobsen SE, Reik W: Genome-wide erasure of DNA methylation in mouse primordial germ cells is affected by AID deficiency. Nature 2010, 463:1101-1105.

22. Rai K, Huggins IJ, James SR, Karpf AR, Jones DA, Cairns BR: DNA demethylation in zebrafish involves the coupling of a deaminase, a glycosylase, and gadd45. Cell 2008, 135:1201-1212.

23. Barreto G, Schafer A, Marhold J, Stach D, Swaminathan SK, Handa V, Doderlein G, Maltry N, Wu W, Lyko F, Niehrs C: Gadd45a promotes epigenetic gene activation by repair-mediated DNA demethylation. Nature 2007, 445:671-675.

24. Niehrs C, Schafer A: Active DNA demethylation by Gadd45 and DNA repair. Trends Cell Biol 2012, 22:220-227.

25. Di Noia JM, Neuberger MS: Immunoglobulin gene conversion in chicken DT40 cells largely proceeds through an abasic site intermediate generated by excision of the uracil produced by AID-mediated deoxycytidine deamination. Eur J Immunol 2004, 34:504-508.

26. Muramatsu M, Kinoshita K, Fagarasan S, Yamada S, Shinkai Y, Honjo T: Class switch recombination and hypermutation require activation-induced cytidine deaminase (AID), a potential RNA editing enzyme. Cell 2000, 102:553-563.

27. Petersen-Mahrt S: DNA deamination in immunity. Immunol Rev 2005, 203:80-97.

28. Morgan HD, Dean W, Coker HA, Reik W, Petersen-Mahrt SK: Activation-induced cytidine deaminase deaminates 5-methylcytosine in DNA and is expressed in pluripotent tissues: implications for epigenetic reprogramming. J Biol Chem 2004, 279:52353-52360.

29. Bestor TH: The DNA methyltransferases of mammals. Hum Mol Genet 2000, 9:2395-2402

30. Kangaspeska S, Stride B, Metivier R, Polycarpou-Schwarz M, Ibberson D, Carmouche RP, Benes V, Gannon F, Reid G: Transient cyclical methylation of promoter DNA. Nature 2008, 452:112-115.

31. Li YQ, Zhou PZ, Zheng XD, Walsh CP, Xu GL: Association of Dnmt3a and thymine DNA glycosylase links DNA methylation with base-excision repair. Nucleic Acids Res 2007, 35:390-400.

32. Metivier R, Gallais R, Tiffoche $C$, Le Peron C, Jurkowska RZ, Carmouche RP, Ibberson D, Barath P, Demay F, Reid G, Benes V, Jeltsch A, Gannon F, Salbert G: Cyclical DNA methylation of a transcriptionally active promoter. Nature 2008, 452:45-50.

33. Gu TP, Guo F, Yang H, Wu HP, Xu GF, Liu W, Xie ZG, Shi L, He X, Jin SG, labal K, Shi YG, Deng Z, Szabo PE, Pfeifer GP, Li J, Xu GL: The role of Tet3 DNA dioxygenase in epigenetic reprogramming by oocytes. Nature 2011, 477:606-610.

34. Wu H, Zhang Y: Tet1 and 5-hydroxymethylation: a genome-wide view in mouse embryonic stem cells. Cell Cycle 2011, 10:2428-2436.

35. Dean W, Santos F, Stojkovic M, Zakhartchenko V, Walter J, Wolf E, Reik W Conservation of methylation reprogramming in mammalian development: aberrant reprogramming in cloned embryos. Proc Natl Acad Sci USA 2001, 98:13734-13738.

36. Inoue A, Zhang Y: Replication-dependent loss of 5hydroxymethylcytosine in mouse preimplantation embryos. Science 2011, 334:194

37. Iqbal K, Jin SG, Pfeifer GP, Szabo PE: Reprogramming of the paternal genome upon fertilization involves genome-wide oxidation of 5methylcytosine. Proc Natl Acad Sci USA 2011, 108:3642-3647.

38. Kobayashi H, Sakurai T, Imai M, Takahashi N, Fukuda A, Yayoi O, Sato S, Nakabayashi K, Hata K, Sotomaru Y, Suzuki Y, Kono T: Contribution of intragenic DNA methylation in mouse gametic DNA methylomes to establish oocyte-specific heritable marks. PLOS Genet 2012, 8:e1002440.

39. Smith ZD, Chan MM, Mikkelsen TS, Gu H, Gnirke A, Regev A, Meissner A: A unique regulatory phase of DNA methylation in the early mammalian embryo. Nature 2012, 484:339-344.

40. Hajkova P, Jeffries SJ, Lee C, Miller N, Jackson SP, Surani MA: Genome-wide reprogramming in the mouse germ line entails the base excision repair pathway. Science 2010, 329:78-82. 
41. Wossidlo M, Arand J, Sebastiano V, Lepikhov K, Boiani M, Reinhardt R, Scholer $\mathrm{H}$, Walter J: Dynamic link of DNA demethylation, DNA strand breaks and repair in mouse zygotes. EMBO J 2010, 29:1877-1888.

42. Teperek-Tkacz M, Pasque V, Gentsch G, Ferguson-Smith AC: Epigenetic reprogramming: is deamination key to active DNA demethylation? Reproduction 2011, 142:621-632

43. Bhutani N, Brady JJ, Damian M, Sacco A, Corbel SY, Blau HM: Reprogramming towards pluripotency requires AID-dependent DNA demethylation. Nature 2010, 463:1042-1047.

44. Schrader CE, Guikema JE, Linehan EK, Selsing E, Stavnezer J: Activation-induced cytidine deaminase-dependent DNA breaks in class switch recombination occur during $\mathrm{G} 1$ phase of the cell cycle and depend upon mismatch repair. J Immunol 2007, 179:6064-6071.

45. Storck S, Aoufouchi S, Weill JC, Reynaud CA: AID and partners: for better and (not) for worse. Curr Opin Immunol 2011, 23:337-344.

46. Clarke HJ: Post-transcriptional control of gene expression during mouse oogenesis. Results Probl Cell Differ 2012, 55:1-21.

47. Parsa JY, Ramachandran S, Zaheen A, Nepal RM, Kapelnikov A, Belcheva A, Berru M, Ronai D, Martin A: Negative supercoiling creates single-stranded patches of DNA that are substrates for AID-mediated mutagenesis. PLoS Genet 2012, 8:e1002518.

48. Boissonneault $\mathrm{G}$ : Chromatin remodeling during spermiogenesis: a possible role for the transition proteins in DNA strand break repair. FEBS Lett 2002, 514:111-114.

49. Chahwan R, Wontakal SN, Roa S: Crosstalk between genetic and epigenetic information through cytosine deamination. Trends Genet 2010, 26:443-448

50. Krokan HE, Bjoras M: Base excision repair. Cold Springs Harb Perspect Biol 2013, 5:a012583.

51. Rada C, Williams GT, Nilsen H, Barnes DE, Lindahl T, Neuberger MS: Immunoglobulin isotype switching is inhibited and somatic hypermutation perturbed in UNG-deficient mice. Curr Biol 2002, 12:1748-1755.

52. Barnes DE, Lindahl T: Repair and genetic consequences of endogenous DNA base damage in mammalian cells. Annu Rev Genet 2004, 38:445-476.

53. Robertson $A B$, Klungland $A$, Rognes $T$, Leiros I: DNA repair in mammalian cells: Base excision repair: the long and short of it. Cell Mol Life Sci 2009, 66:981-993.

54. Cortazar D, Kunz C, Selfridge J, Lettieri T, Saito Y, MacDougall E, Wirz A Schuermann D, Jacobs AL, Siegrist F, Steinacher R, Jiricny J, Bird A, Schar P: Embryonic lethal phenotype reveals a function of TDG in maintaining epigenetic stability. Nature 2011, 470:419-423.

55. Nilsen H, Rosewell I, Robins P, Skjelbred CF, Andersen S, Slupphaug G, Daly G, Krokan HE, Lindahl T, Barnes DE: Uracil-DNA glycosylase (UNG)deficient mice reveal a primary role of the enzyme during DNA replication. Mol Cell 2000, 5:1059-1065

56. Chen CC, Wang KY, Shen CK: DNA 5-methylcytosine demethylation activities of the mammalian DNA methyltransferases. $J$ Biol Chem 2013, 288:9084-9091.

57. Ficz G, Hore TA, Santos F, Lee HJ, Dean W, Arand J, Krueger F, Oxley D, Paul YL, Walter J, Cook SJ, Andrews S, Branco MR, Reik W: FGF signaling inhibition in ESCS drives rapid genome-wide demethylation to the epigenetic ground state of pluripotency. Cell Stem Cell 2013, 13:351-359.

58. Kaneda M, Hirasawa R, Chiba H, Okano M, Li E, Sasaki H: Genetic evidence for Dnmt3a-dependent imprinting during oocyte growth obtained by conditional knockout with Zp3-Cre and complete exclusion of Dnmt3b by chimera formation. Genes Cells 2010 [Epub ahead of print].

59. Shirane K, Toh H, Kobayashi H, Miura F, Chiba H, Ito T, Kono T, Sasaki H: Mouse oocyte methylomes at base resolution reveal genome-wide accumulation of non-CpG methylation and role of DNA methyltransferases. PLoS Genet 2013, 9:e1003439.

60. Wood RD, Mitchell M, Sgouros J, Lindahl T: Human DNA repair genes. Science 2001, 291:1284-1289.

61. Derijck A, van der Heijden G, Giele M, Philippens M, de Boer P: DNA double-strand break repair in parental chromatin of mouse zygotes, the first cell cycle as an origin of de novo mutation. Hum Mol Genet 2008, 17:1922-1937.

62. Kaneda M, Okano M, Hata K, Sado T, Tsujimoto N, Li E, Sasaki H: Essential role for de novo DNA methyltransferase Dnmt3a in paternal and maternal imprinting. Nature 2004, 429:900-903.
63. Lewandoski M, Wassarman KM, Martin GR: Zp3-cre, a transgenic mouse line for the activation or inactivation of loxP-flanked target genes specifically in the female germ line. Curr Biol 1997, 7:148-151.

64. Tahiliani M, Koh KP, Shen Y, Pastor WA, Bandukwala H, Brudno Y, Agarwal S, lyer LM, Liu DR, Aravind L, Rao A: Conversion of 5-methylcytosine to 5-hydroxymethylcytosine in mammalian DNA by MLL partner TET1. Science 2009, 324:930-935.

65. Probst AV, Santos F, Reik W, Almouzni G, Dean W: Structural differences in centromeric heterochromatin are spatially reconciled on fertilisation in the mouse zygote. Chromosoma 2007, 116:403-415.

66. Patenaude AM, Di Noia JM: The mechanisms regulating the subcellular localization of AID. Nucleus 2010, 1:325-331.

doi:10.1186/1756-8935-6-39

Cite this article as: Santos et al: Active demethylation in mouse zygotes involves cytosine deamination and base excision repair. Epigenetics \& Chromatin 2013 6:39

\section{Submit your next manuscript to BioMed Central and take full advantage of:}

- Convenient online submission

- Thorough peer review

- No space constraints or color figure charges

- Immediate publication on acceptance

- Inclusion in PubMed, CAS, Scopus and Google Scholar

- Research which is freely available for redistribution 